\title{
WEB ALERT
}

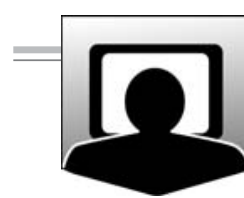

\section{Editor Andreia Biolo, MD}

Hospital de Clínicas de Porto Alegre, Rua Ramiro Barcelos 2350,

Porto Alegre/RS, CEP 90035-003, Brazil.

E-mail: abiolo@hcpa.ufrgs.br

\section{Heart Failure Online}

http://www.heartfailure.org

This is an interesting site that is sponsored in part by the San Diego Cardiac Center and the Sharp Foundation for Cardiovascular Research and Education. The site has five main sections, which include information about 1) the heart, 2) heart failure, 3) prevention, 4) treatment, and 5) recent news reports. The site provides easy access to information, with informative diagrams about the cardiocirculatory system and changes present in heart failure, including explanations on the pathophysiology of primary heart failure signs and symptoms. It also provides information on interventions and their benefits for patients with heart failure.

\section{The Failing Heart}

http://www.cme-heartfailure.net

This is a valuable educational site that provides access to internationally focused medical information on heart failure, specifically for health care professionals. Registration for the site is free and includes access to several interesting topics on heart failure pathology, diagnosis, and treatment. Several continuing medical education interactive learning courses are also available, covering focused topics on heart failure, such as diabetes in heart failure and evaluation and management of diastolic heart failure.

\section{Atlas of Echocardiography}

http://www.med.yale.edu/intmed/cardio/echo_atlas

The Atlas of Echocardiography is part of the cardiothoracic imaging site from Yale University. In addition to the atlas, the site provides sections on anatomy, imaging technology, image findings, as well as a case index. The contents include transthoracic and transesophageal views, ventricular function, disease entities (eg, several valvuloplasties, cardiomyopathy, and ischemia), and references for basic terminology. The images are of excellent quality, and each topic provides background information, schematic and echocardiographic views, animations, and three-dimensional models. It is a valuable educational site for those interested in learning or reviewing echocardiographic imaging. 